\title{
Des pharmas éthiques? Responsabilité et contrôles lors d'essais cliniques
}

\section{Jacques Schiltknecht}

Je tiens à remercier le Dr Etzel Gysling pour sa relecture critique du manuscrit et pour ses remarques pertinentes.

Une prise de position de l'industrie pharmaceutique suisse pratiquant la recherche sera publiée dans le prochain numéro du BMS.

Correspondance: Dr Jacques Schiltknecht Dreilindenstrasse 52 CH-6006 Lucerne Tél. 0414205505

bj.schiltknecht[at]bluewin.ch
«Dans la recherche médicale, le devoir du médecin est de protéger la vie, la santé, la dignité et l'intimité de la personne.»

(Déclaration d'Helsinki de l'Association Médicale Mondiale, 2000)

A la mi-septembre 2013, la Déclaration de Berne (DB) publiait un dossier spécial avec un titre provocateur: «Des cobayes humains au rabais». L'ONG y dénonce de nombreux exemples de manquements éthiques survenus lors d'essais cliniques menés par des entreprises pharmaceutiques. Des tendances inquiétantes, dont j'étais loin de me douter, sont consignées dans les 23 pages du dossier, avec documents, statistiques et graphiques à l'appui.

J'étais embarrassé de constater que, durant mes 40 années de pratique en tant que médecin généraliste, je n'avais que rarement pensé aux êtres humains qui mettent leur corps à la disposition de la médecine dans le cadre d'essais cliniques. Pris par le quotidien de mon cabinet, je ne faisais que peu de cas d'articles critiques, comme ceux par exemple d'Etzel Gysling dans la revue «Pharma-Kritik».

Il m'a fallu rechercher des termes comme Déclaration d'Helsinki, CIOMS et OClin sur internet. Et je me suis rapidement rendu compte, en parlant avec des amis médecins, que je n'étais pas seul dans ce cas. J'ignorais, par exemple, que de nombreux essais cliniques ne sont pas menés directement par les firmes pharmaceutiques mais sont confiés à des «Contract Research Organizations» (CRO). J'ignorais également que la moitié ou presque des essais cliniques a lieu dans des pays qui connaissent une grande pauvreté et/ou de fréquentes violations des droits humains, comme la Russie, la Chine, le Mexique, l'Argentine et bien d'autres.

Pourquoi les tests sont-ils de plus en plus souvent délocalisés dans ces pays?

Selon scienceINDUSTRIES, l'organe faîtier des entreprises pharmaceutiques, les essais cliniques représentent deux tiers des coûts nécessaires à la mise sur le marché d'un nouveau médicament. Or, écourter la phase de recherche clinique permet d'allonger d'autant la lucrative période de commercialisation d'un médicament protégé par un brevet.

Selon la DB, les entreprises pharmaceutiques sont intéressées à mener leurs essais dans les pays susmentionnés pour plusieurs raisons: organes de contrôle laxistes, autorisations plus faciles à obtenir, commissions d'éthique peu regardantes, recrutement beaucoup plus rapide de sujets issus de couches pauvres et peu éduquées. De plus, en cas de problème, les personnes issues de ces milieux engagent rarement des poursuites pour obtenir des dédommagements.

Les représentants de l'industrie pharmaceutique insistent sur la nécessité de respecter les normes éthiques afin de prévenir les abus. Selon eux, le respect serait garanti par des «surveillants» ou «Clinical

\section{«Organes de contrôle laxistes, autori- sations plus faciles à obtenir, commis- sions d'éthique peu regardantes...»}

Research Associates», à qui l'on confie la supervision des essais cliniques dans les pays à risque, et qui sont payés par les «promoteurs». Des audits seraient menés de façon régulière, et le rapport final ferait état du respect des normes éthiques (un rapport qui reste cependant interne).

Les raisons mises en avant par l'industrie pharmaceutique pour justifier les tests menés dans des pays émergents diffèrent sensiblement de celles de la DB: d'abord, il ne s'agirait pas de faire des économies, mais de tester les médicaments des personnes d'ethnies et de cultures différentes, dans des pays variés comme la Chine, la Russie, l'Inde, l'Ethiopie ou Taïwan. Ensuite, il faudrait, pour obtenir une autorisation de mise sur le marché dans ces pays, que les tests aient été menés sur des populations locales. La prévalence des maladies jouerait enfin également un rôle, par exemple dans le cas de la malaria.

Pour la Déclaration de Berne, le respect des standards éthiques dans le sens de la déclaration d'Helsinki devrait être vérifié par Swissmedic avant que le médicament soit autorisé en Suisse. Ce qui impliquerait que les rapports d'essais cliniques et les comptes rendus du «surveillant» soient mis à la disposition de l'agence.

La volonté manifeste des compagnies pharmaceutiques de faire respecter à l'interne leur code en matière d'éthique ainsi que leur politique de transparence est certes louable. Il faudrait cependant aussi que ces firmes acceptent de se laisser contrôler par une instance indépendante et rendent des comptes à Swissmedic ainsi qu'à l'opinion publique.

Leurs engagements éthiques doivent être accessibles et exprimés de façon simple et claire. 
Pour lancer la discussion, je souhaite évoquer quelques principes, qui sont en partie tirés des engagements formels des entreprises et de divers codes internationaux:

- Toutes les études cliniques doivent être annoncées et enregistrées de façon centralisée et systématique. Les données de toute étude initiée doivent être mises à la disposition de l'agence du médicament concernée (Swissmedic, EMA, FDA, etc.) dans leur totalité et de façon transparente.

- Les résultats de l'étude doivent être intégralement publiés. Une fois les médicaments mis sur le marché, ils doivent être à la disposition de tous.

- Les falsifications et la dissimulation de données négatives ou de séquences complètes d'essais doivent être sanctionnés et faire l'objet de poursuites judiciaires. Les «lanceurs d'alerte» ou «Whistleblower» ne doivent en aucun cas être inquiétés.

- Les promoteurs doivent s'assurer, en particulier dans les pays où le contrôle étatique est insuffisant, que les personnes nommées dans les commissions d'éthique soient compétentes et intègres. Les entreprises doivent vérifier d'ellesmêmes que le travail des commissions d'éthique et le déroulement des essais cliniques soient conformes à la «bonne pratique clinique» (Good Clinical Practice, GCP) reconnue au niveau international. Un rapport concernant ces éléments doit être remis à Swissmedic (ainsi qu'aux autres autorités concernées).

- Le déroulement de l'essai doit être expliqué de manière précise et dans leur langue maternelle à tous les participants, et le contrat qui leur est soumis doit être absolument clair. Un service de médiation doit être disponible si les participants ont des questions ou des problèmes, et ils doivent avoir la possibilité de recourir devant la justice le cas échéant.

- Les personnes issues de milieux pauvres qui, dans le cadre de l'essai, ont peut-être bénéficié pour la première fois d'un traitement adéquat, doivent y avoir accès même une fois l'essai terminé.

Articles interactifs

Vous souhaitez commenter cet article? II vous suffit pour cela d'utiliser la fonction «Ajouter un commentaire» dans la version en ligne. Vous pouvez également consulter les remarques de vos confrères sous: www.bullmed.ch/ numero-actuel/ articles-interactifs/
- «Les compagnies pharmaceutiques doivent respecter l'esprit et la lettre des codes pertinents de l'industrie. Pour ce faire, les compagnies pharmaceutiques veilleront à ce que tous les membres concernés de leurs personnels reçoivent une formation appropriée.» (Extrait du Code de l'IFPMA sur les bonnes pratiques)

Alors que la pression pour plus d'innovation est grande, il faut du courage pour garantir le respect des principes éthiques. Les dispositions légales, les engagements formels comme le Code pharmaceutique de l'industrie suisse ainsi que les contrôles sont néces- saires et utiles, mais rien ne remplace le respect et l'empathie. Serions-nous prêts à participer à un essai clinique pour le progrès de l'humanité? Est-ce que nous laisserions nos enfants participer à de tels tests? Nous devons avoir le courage de nous poser ce genre de questions.

Respecter scrupuleusement les principes de bonne pratique clinique (GCP) exige des moyens et de l'énergie, mais c'est aussi une question stratégique. N'est-ce pas, à long terme, un excellent si ce n'est le meilleur investissement possible?

Il est démontré que cultiver l'éthique en son sein représente un atout majeur pour une entreprise. Par ce biais, le personnel est encouragé à participer et peut s'identifier pleinement aux buts de l'entreprise. Cela diminue également les accidents et la tendance à l'absentéisme, sans parler de sa valeur inestimable pour la réputation de l'entreprise. A l'inverse, des incitatifs financiers exorbitants attisent l'appât du gain et entraînent une concurrence parfois malsaine à l'interne; elle pousse les managers au burnout et démoralise l'entier du personnel jusqu'aux simples collaborateurs, qui sont à la base de tout succès d'entreprise.

Il s'agit ici de trouver le bon équilibre des valeurs. D'un côté, les actionnaires veulent voir leur profit augmenter avec le cours de la Bourse, ce qui est légitime dans une certaine mesure. De l'autre, les clients, l'opinion publique et d'autres acteurs sont intéressés par le produit: des médicaments efficaces, testés consciencieusement et dans le respect de l'éthique.

Ces deux intérêts doivent être considérés, mais la dignité des personnes participant à l'essai doit primer sur tous les autres. L'éthique dans le cadre de la recherche pharmaceutique devrait être une évidence, sans qu'il y ait besoin de recourir, ici aussi, à un label spécifique.

La campagne de la DB est un pavé dans la mare, et nous n'avons pas fini d'en entendre parler. Il faut espérer que nous n'assistions pas à un incessant ballet stérile de démentis et d'accusations, où chacun se renvoie la responsabilité. Les firmes pharmaceutiques doivent faire preuve d'initiative et d'une éthique de la responsabilité - au sens où l'entendait Max Weber - afin que la culture d'entreprise dans la branche s'en retrouve renforcée. Leurs dirigeants doivent s'assurer de manière plus intensive encore du strict respect des standards éthiques découlant de la Déclaration d'Helsinki.

A nous autres, médecins, d'être attentifs et d'intervenir si nécessaire!

Les critiques, apports, propositions et précisions sont les bienvenus, en particulier en provenance de l'industrie pharmaceutique.

Bibliographie sur demande - la plupart des informations sont accessibles facilement sur Internet. 